\title{
Conformal/Poincaré Coset, cosmology, and descendants of Lovelock terms
}

\author{
Gregory Gabadadze and Giorgi Tukhashvili॰ \\ Center for Cosmology and Particle Physics, Department of Physics, New York University, \\ 726 Broadway, New York, New York 10003, USA
}

(Received 20 May 2020; accepted 3 July 2020; published 17 July 2020)

\begin{abstract}
We calculate six invariant terms of a gravitational field theory that nonlinearly realizes the conformal/ Poincare quotient and reduce to the known conformal Galileons in the limit when only the conformal mode is kept. Five of the six terms are regular coset terms, while the sixth is a Wess-Zumino (WZ) term that gives the well-known gravitational action for the trace anomaly. The obtained terms can be embedded in a quantum effective field theory (EFT) without spoiling their key features, although at a cost of certain finetunings. The additional massive modes that appear in the EFT would have been troublesome, however, for sub-Planckian curvatures their masses are (super)-Planckian, and therefore the respective states are outside of the EFT regime. We discuss certain novel cosmological solution of this theory and their validity within the EFT. Furthermore, we show that the obtained 4D terms, except the WZ term, can also be derived from higher dimensional Lovelock terms by reducing the latter to the genuinely four-dimensional terms according to a well-defined algorithm.
\end{abstract}

DOI: $10.1103 /$ PhysRevD.102.024054

\section{INTRODUCTION AND SUMMARY}

Theories that modify gravity in the infrared, such as brane-induced gravity [1] or massive gravity [2,3], also exhibit interesting ultraviolet properties as quantum effective field theories: the terms that amend the Einstein-Hilbert (EH) action in these theories-e.g., the covariant graviton mass term-turn out to contain special higher dimensional (irrelevant) operators at a scale much below the Planck mass; while the emergence of this strong interaction scale is not unlike in massive non-Abelian gauge theories [4], both the dynamics and open questions due to these higher dimensional operators in massive gravity are considerably richer, see, e.g., [5-15].

These irrelevant operators describe nonlinear interactions of helicity- 1 and helicity- 0 components of a massive spin-2 graviton. In particular, the helicity-0 interactions are described by the so-called Galileon field theories $[8,16]$.

In the absence of gravity the Galileon is an interesting effective field theory by its own. If one were to start with a Galileon as a stand-alone scalar field theory without gravity, then we know that massive gravity would provide gravitational dressing of the Galileon. In the dressed theory the Galileon appears as a gauge mode of a tensor field.

Published by the American Physical Society under the terms of the Creative Commons Attribution 4.0 International license. Further distribution of this work must maintain attribution to the author(s) and the published article's title, journal citation, and DOI. Funded by SCOAP .
In more physical terms, the Galileon particle is a NambuGoldstone (NG) mode that is being absorbed as a longitudinal mode by a massive spin-2 state. ${ }^{1}$ Then, nonlinear interactions of the longitudinal model are restricted severely by the requirement for the theory to propagate only 5 physical degrees of freedom of a massive spin- 2 on an arbitrary background, and this requirement selects the Galileons [2,3,18].

There is another interesting class of derivatively interacting scalar field theories, the conformal Galileons [16]. They have been used in cosmology to describe alternatives for the early Universe [19-21], where the gravity was introduced through direct covariantization of the conformal Galileon action, with the total action containing the physical conformal Galileon field alongside with a massless graviton.

It seems natural to ask [22] whether there exists a gravitational field theory where the conformal Gallileon would be a NG mode (gauge mode), like the ordinary Galileon is in massive gravity.

This question was addressed in [22] in 3D. The approach used the observation that the conformal Galilleon terms emerge as coset and Wess-Zumino (WZ) terms in a nongravitational theory, with the conformal group spontaneously broken to the Poincaré subgroup [23]. To obtain the gravitationally dressed conformal Galileons we considered a full gravitational conformal/Poincaré coset in 3D.

\footnotetext{
${ }^{1}$ Note that this is different from the direct covariantization of the Galileon [17], where the Galileon is an independent physical scalar degree of freedom coupled to gravity.
} 
This led to the well-known action of New Massive Gravity (NMG) [24] and its generalization [25], both obtained in a local Weyl invariant form. The conformal Galileon itself emerged as a NG mode of the broken scale invariance and as a gauge mode of the local Weyl symmetry.

The next natural question appears to be this: what is a 4D gravitational theory that contains conformal Galileons as NG/gauge modes? This seems to be a worthy question to address since massive gravity cannot give an answer to it, and a $4 \mathrm{D}$ generalization of NMG is not known. Besides, the sought theory might be expected to inherit some interesting cosmological solutions of the conformal Galileon.

In the present work we will build such a 4D theory using the coset construction. The coset will give us six special terms: the first one is the Weyl invariant version of the cosmological constant, the second is the Weyl invariant EH term, the third one is the Gauss-Bonnet (GB) term, the fourth is a special combination of invariant cubic curvature terms, the fifth is a special combination of invariant quartic curvature terms, and, the sixth one emerges as a WZ term describing the action for the conformal anomaly [26] (see also [27]). All these terms, except the fourth and fifth, are well known. Their defining feature is that their conformal mode exactly reproduces all the known conformal Galileons. Hence, the equations of motion of the theory-once reduced to the conformal mode-are necessarily of the second order. Since the conformal mode is a scale factor in homogeneous and isotropic cosmology, this theory gives novel, potentially viable cosmological solutions, as we will show.

The obtained action should be viewed as part of a certain quantum effective field theory (EFT) with an infinite number of other terms. The EFT however, can only contain additional terms proportional to at least one power of the Weyl tensor to preserve the above described properties. This requires fine-tunings of the counterterms in the standard renormalization procedure. The obtained theory would harbor additional degrees of freedom due to the higher derivative terms, but their masses are at or above the cutoff of EFT for reasonable values of other parameters. Thus, the theory albeit being fine-tuned, is otherwise a good EFT. ${ }^{2}$

Last but not least, we show that five out of the six new terms can be obtained from the higher dimensional Lovelock terms via certain dimensional reduction across various dimensions. Only the sixth, the WZ term, can not be obtained through this procedure, since it is not Weyl invariant.

Interestingly, D. Glavan and Chunshan Lin [28] proposed a certain continuation of the 5D GB term to fourdimensions. The obtained terms have their merits, however, are not truly four dimensional $[29,30]$. Our procedure bears

\footnotetext{
${ }^{2}$ Fine-tuning without symmetry is viewed as a deficiency by the authors.
}

a formal resemblance to that of [28], but is both conceptually and technically different: the reduction of the Lovelock terms we perform gives local well-defined terms in lower dimensions; for instance, the 5D GB term reduces to a 3D local term of NMG, while the 6D Lovelock term to a pure 4D term belonging to the coset, as shown in Sec. V.

Throughout the paper we use the following conventions and notations: $\delta_{\nu_{1} \cdots \nu_{n}}^{\mu_{1} \cdots \mu_{n}}=\delta_{\nu_{1}}^{\mu_{1}} \cdots \delta_{\nu_{n}}^{\mu_{n}} \pm$ permutations. $D$ stands for both, the generator of dilatations and covariant derivative. The Planck mass will be set to one, unless it is shown explicitly. The sign, $\simeq$, means "equals up to a total derivative." The Riemann tensor is $R^{\rho}{ }_{\mu \sigma \nu}=\partial_{\sigma} \Gamma_{\mu \nu}^{\rho}+\cdots$. $R_{\mu \nu}^{n+1} \equiv R_{\mu}^{\rho_{1}} R_{\rho_{1}}^{\rho_{2}} \cdots R_{\rho_{n} \nu}$ and $\left[R^{n}\right] \equiv R_{\rho_{2}}^{\rho_{1}} R_{\rho_{3}}^{\rho_{2}} \cdots R_{\rho_{1}}^{\rho_{n}}$. The total derivatives for the helicity- 0 mode start with the terms normalized as $L_{n}^{T D}=\left(\partial^{2} \pi\right)^{n}+\cdots$. The Schouten tensor is normalized as follows: $S_{\nu}^{\mu}=\frac{1}{n-2}\left(R_{\nu}^{\mu}-\frac{R}{2(n-1)} \delta_{\nu}^{\mu}\right)$.

\section{II. $S O(n, 2)$ BROKEN TO ISO $(n-1,1)$}

Let us briefly summarize the coset formalism [31] adopted for the conformal group [32,33]. One starts in $n$ space-time dimensions and postulates the conformal group to be spontaneously broken down to its Poincaré subgroup. Conformal algebra in $n$ dimensions is realized by the $n(n+1) / 2$ Poincaré generators $\left(P_{a}, J_{a b}\right)$, plus $n$ generators of special conformal transformations, $K_{a}$, plus one generator of dilatations, $D$. These generators satisfy the following standard commutation relations:

$$
\begin{gathered}
{\left[P_{a}, D\right]=P_{a}, \quad\left[D, K_{a}\right]=K_{a},} \\
{\left[J_{a b}, K_{c}\right]=\eta_{a c} K_{b}-\eta_{b c} K_{a}, \quad\left[K_{a}, P_{b}\right]=2 J_{a b}-2 \eta_{a b} D} \\
{\left[J_{a b}, P_{c}\right]=\eta_{a c} P_{b}-\eta_{b c} P_{a},} \\
{\left[J_{a b}, J_{c d}\right]=\eta_{a c} J_{b d}-\eta_{b c} J_{a d}+\eta_{b d} J_{a c}-\eta_{a d} J_{b c}}
\end{gathered}
$$

Since $\left[K_{a}, P_{b}\right] \propto-2 \eta_{a b} D$ not all the NG fields of the coset are independent; the inverse Higgs constraint (IHC) [32] will enable us to eliminate the NG's related to broken $K_{a}$ 's in favor of the NG related to broken $D$.

\section{A. Flat space-time}

As a warm-up we start with a flat space-time metric, $\eta_{\mu \nu}$. A convenient parametrization for the $S O(n, 2) / I S O$ $(n-1,1)$ coset element is given by

$$
\Sigma=e^{\pi D} e^{\xi^{a} K_{a}} .
$$

One can construct the Maurier-Cartan one-form as follows:

$\Sigma^{-1}\left(d+\delta^{a} P_{a}\right) \Sigma=E^{a} P_{a}+\omega_{K}^{a} K_{a}+\omega_{D} D-\frac{1}{2} \omega_{J}^{a b} J_{a b}$. 
Here $\delta^{a} \equiv \delta_{\mu}^{a} d x^{\mu}$, and the extra piece in the parenthesis on the left-hand side (lhs) is introduced for further convenience (it can be traded for an extra factor of $e^{x^{a} P_{a}}$ in the coset element in an equivalent approach). The expressions for the one forms in front of the generators on the rhs can be calculated using conformal algebra given above,

$$
\begin{gathered}
E^{a}=e^{\pi} \delta^{a}, \\
\omega_{D}=d \pi+2 E^{a} \xi_{a}, \\
\omega_{K}^{a}=d \xi^{a}-\xi^{2} E^{a}+\xi^{a} \omega_{D}, \\
\omega_{J}^{a b}=-2 E^{a} \xi^{b}+2 E^{b} \xi^{a} .
\end{gathered}
$$

Here $\xi^{a}$ is a zero form Lorentz vector. We make a distinction between the Latin and Greek indices in preparation to introduce a nontrivial metric for a general pseudoRiemannian space-time manifold; the Latin indices will be used for tangent space-times.

\section{B. Dynamical metric}

To covariantize the coset in the first order formalism we gauge the translations and rotations [34]. To gauge translations we simply replace $\delta^{a}$ by the field $e^{a}$ on the lhs of (2.5), but for gauging rotations an extra piece is needed,

$$
\begin{aligned}
& \Sigma^{-1}\left(d+e^{a} P_{a}-\frac{1}{2} \omega^{a b} J_{a b}\right) \Sigma \\
& \quad=E^{a} P_{a}+\Omega_{K}^{a} K_{a}+\Omega_{D} D-\frac{1}{2} \Omega_{J}^{a b} J_{a b} .
\end{aligned}
$$

A straightforward calculation leads to

$$
\begin{gathered}
E^{a}=e^{\pi} e^{a}, \\
\Omega_{D}=D \pi+2 E^{a} \xi_{a}, \\
\Omega_{K}^{a}=D^{a}-\xi^{2} E^{a}+\xi^{a} \Omega_{D}, \\
\Omega_{J}^{a b}=\omega^{a b}-2 E^{a} \xi^{b}+2 E^{b} \xi^{a} .
\end{gathered}
$$

Here the gauge field $e^{a}$ can be interpreted as an n-bein and $\omega^{a b}$ as a spin connection of pure spin-2 field, $D$ corresponds to covariant derivative with respect to $\omega^{a b}$, i.e., $D=d+\omega$. Since we are working with a dynamical pseudo-Riemannian manifold it is natural to construct the curvature two form,

$$
\begin{aligned}
\mathcal{R}^{a b}= & d \Omega_{J}^{a b}+\Omega_{J}^{a c} \wedge \Omega_{J c}{ }^{b} \\
= & R^{a b}+2 E^{a} \wedge \Omega_{K}^{b}+2 \Omega_{K}^{a} \wedge E^{b}, \\
& R^{a b}=d \omega^{a b}+\omega^{a c} \wedge \omega_{c}{ }^{b} .
\end{aligned}
$$

In the next section we will use some of these elements, but not all, to build an effective action.

\section{4D CONFORMAL GALILEONS AND THEIR EMBEDDING}

The goal of this section is to show that the 4D conformal Galileon describes a theory of the conformal mode of a diffeomorphism invariant gravitational theory, made out of the conformal/Poincaré coset and WZ terms.

\section{A. Flat background metric}

Reference [23] showed that the flat space conformal Galileons can be constructed using the flat space conformal/Poincare coset. For convenience we will briefly summarize this remarkable result below before introducing the gravitational field in the next subsection.

Among the three one-forms in (2.6)-(2.8) only two carry a single local Lorentz index. Therefore only those terms, (2.6) and (2.8), can be used to construct the four-form actions without invoking additional covariant derivatives,

$$
\begin{aligned}
& A_{0}=\int_{\mathcal{M}_{4}} \varepsilon_{a b c d} E^{a} \wedge E^{b} \wedge E^{c} \wedge E^{d}, \\
& A_{1}=\int_{\mathcal{M}_{4}} \varepsilon_{a b c d} E^{a} \wedge E^{b} \wedge E^{c} \wedge \omega_{K}^{d}, \\
& A_{3}=\int_{\mathcal{M}_{4}} \varepsilon_{a b c d} E^{a} \wedge \omega_{K}^{b} \wedge \omega_{K}^{c} \wedge \omega_{K}^{d}, \\
& A_{4}=\int_{\mathcal{M}_{4}} \varepsilon_{a b c d} \omega_{K}^{a} \wedge \omega_{K}^{b} \wedge \omega_{K}^{c} \wedge \omega_{K}^{d} .
\end{aligned}
$$

We left out $\varepsilon_{a b c d} E^{a} \wedge E^{b} \wedge \omega_{K}^{c} \wedge \omega_{K}^{d}$, since it is a total derivative.

In addition to the above coset terms, there are WessZumino terms [23]: they appear as pullbacks of certain fiveforms on a four-dimensional hypersurface. Straightforward calculations show that only one of the possible five WZ terms is independent of the coset terms already accounted above. This independent term reads as follows:

$$
A_{2} \equiv \int_{\mathcal{M}_{5}} \varepsilon_{a b c d} \omega_{D} \wedge E^{a} \wedge E^{b} \wedge \omega_{K}^{c} \wedge \omega_{K}^{d}
$$

The above expression for $A_{2}$ can be written more explicitly as follows:

$$
\begin{aligned}
A_{2}= & \int_{\mathcal{M}_{4}} \varepsilon_{a b c d}\left(\frac{1}{2} E^{a} \wedge E^{b} \wedge d \xi^{c} \wedge d \xi^{d}-\frac{2}{3} \xi^{2} E^{a} \wedge E^{b}\right. \\
& \left.\wedge E^{c} \wedge d \xi^{d}+\frac{1}{4} \xi^{4} E^{a} \wedge E^{b} \wedge E^{c} \wedge E^{d}\right) .
\end{aligned}
$$

As already noted in the previous section, due to the commutator $\left[K_{a}, P_{b}\right] \propto-2 \eta_{a b} D$, not all the NG fields of 
the coset are independent; the inverse Higgs constraint (IHC) [32] can be invoked to eliminate $\xi^{a}$, related to broken $K_{a}$, in favor of a derivative of $\pi$, related to broken $D$. Conventionally, this is done by imposing $\omega_{D}=0$; in our case, the IHC is a solution of the equations of motion for $\xi^{a}$ [35] that also satisfies $\omega_{D}=0$,

$$
\delta_{\xi} A_{n}=0, \quad n=0,1,2,3,4 .
$$

Hence, we use IHC to express $\xi^{\mu}=-1 / 2 e^{-\pi} \partial^{\mu} \pi$, and substitute this into the actions $A_{n}, n=1,2,3,4$, which then reduce to the conformal Galileons,

$$
\begin{gathered}
A_{0}=\int d^{4} x e^{4 \pi} \\
A_{1} \simeq \int d^{4} x e^{2 \pi}(\partial \pi)^{2} \\
A_{2} \simeq \int d^{4} x(\partial \pi)^{2}\left(L_{1}^{T D}+\frac{1}{2}(\partial \pi)^{2}\right) \\
A_{3} \simeq \int d^{4} x e^{-2 \pi}(\partial \pi)^{2}\left(L_{2}^{T D}-\frac{1}{2}(\partial \pi)^{2} L_{1}^{T D}+\frac{1}{2}(\partial \pi)^{4}\right) \\
A_{4} \simeq \int d^{4} x e^{-4 \pi}(\partial \pi)^{2} \\
\times\left(L_{3}^{T D}-3(\partial \pi)^{2} L_{2}^{T D}+5(\partial \pi)^{4} L_{1}^{T D}-\frac{11}{4}(\partial \pi)^{6}\right) .1
\end{gathered}
$$

As already mentioned, $L_{n}^{T D}$ denote the total derivative terms made of $\pi$ with the convention that $(\square \pi)^{n}$ enters with the unit coefficient, e.g., $L_{1}^{T D}=\square \pi, L_{2}^{T D}=(\square \pi)^{2}+\ldots$, etc. Moreover, each term in $A_{n}$ contains $2 n$ derivatives, but the number of fields is different in each of them. Each $A_{n}$ is invariant with respect to the conformal Galilean transformations (consisting of the linearly realized Poincaré transformations, and nonlinearly realized special conformal transformations and dilatations of $\pi$ ). Equations of motion for the Galileons have at most two time derivatives acting per field.

\section{B. Gravitational dressing of 4D conformal Galileons}

Any local flat-space CFT must be Weyl invariant after it is embedded covariantly in curved space-time [36]. ${ }^{3}$ Thus, we expect gravitationally dressed conformal Galileons to

\footnotetext{
${ }^{3}$ One could of course break explicitly Weyl symmetry by adding some breaking terms as long as they vanish in the flat space limit; however, we will not be including such terms and will preserve Weyl invariance in the classical action.
}

be Weyl invariant. The Weyl transformations of the relevant fields are [22]

$$
\begin{gathered}
e^{a} \rightarrow e^{\sigma} e^{a}, \\
\pi \rightarrow \pi-\sigma, \\
\xi^{a} \rightarrow \xi^{a}+\frac{1}{2} e^{-\pi} \partial^{a} \sigma .
\end{gathered}
$$

Only three of the building blocks found in the previous section are invariant under these transformations: the one forms $E^{a}$ and $\Omega_{D}$, and the two-form $\mathcal{R}^{a b}$.

It is instructive to recall how the construction works in a simpler, 3D case [22]: the Weyl tensor vanishes identically in $3 \mathrm{D}$, and one can use the Schouten tensor, $\mathcal{S}^{a}$, instead of the curvature two-form, $\mathcal{R}^{a b}$. Then, out of the three oneforms $E^{a}, \Omega_{D}$, and $\mathcal{S}^{a}$, one can build four three-form actions for the 3D Galileons [22].

In $4 \mathrm{D}$ the construction is more involved, the Weyl tensor is no longer zero, and one has to use the curvature two form, $\mathcal{R}^{a b}$, as a building block. In addition, one can also define the Weyl invariant curvature one-form, $\mathcal{R}^{a}$, and a zero-form, the Ricci scalar $\mathcal{R}$, by using the interior product,

$$
\begin{aligned}
\mathcal{R}^{a} & =i_{E_{a}} \mathcal{R}^{a b}=e^{-\pi} R^{b}+4 \Omega_{K}^{b}+2 \Omega_{K} E^{b}, \\
\mathcal{R} & =i_{E_{b}} i_{E_{a}} \mathcal{R}^{a b}=e^{-2 \pi} R+12 \Omega_{K} .
\end{aligned}
$$

Here $E_{a}=E_{a}^{\mu} \partial_{\mu}$, with $E_{a}^{\mu} E_{\mu}^{b}=\delta_{a}^{b}$ and $\Omega_{K} \equiv i_{E_{a}} \Omega_{K}^{a}$.

Let us now build the action. At the level of zero derivatives we can write down only one term,

$$
\int_{\mathcal{M}_{4}} \varepsilon_{a b c d} E^{a} \wedge E^{b} \wedge E^{c} \wedge E^{d}
$$

This is the Weyl invariant version of the cosmological constant. In the unitary gauge, where $\pi=0$, and in the metric formalism,

$$
\mathcal{A}_{0} \equiv \int d^{4} x \sqrt{g}
$$

At the level of two derivatives we can write down three invariants, but only one of them is independent,

$$
\int_{\mathcal{M}_{4}} \varepsilon_{a b c d} \mathcal{R}^{a b} \wedge E^{c} \wedge E^{d}
$$

This is a Weyl invariant extension of the Einstein-Hilbert (EH) term; Weyl symmetry can be fixed by the unitary gauge, $\pi=0$, reducing $\mathcal{A}_{1}$ to the $\mathrm{EH}$ term,

$$
\mathcal{A}_{1} \equiv \int d^{4} x \sqrt{g} R
$$


(however one should keep in mind the Weyl anomaly, which we will discuss at the end of this section).

Furthermore, at the level of four derivatives there are three independent invariants,

$$
\begin{aligned}
& \int_{\mathcal{M}_{4}} \varepsilon_{a b c d} \mathcal{R}^{a b} \wedge \mathcal{R}^{c d}, \quad \int_{\mathcal{M}_{4}} \varepsilon_{a b c d} \mathcal{R}^{a} \wedge \mathcal{R}^{b} \wedge E^{c} \wedge E^{d}, \\
& \int_{\mathcal{M}_{4}} \varepsilon_{a b c d} E^{a} \wedge E^{b} \wedge E^{c} \wedge E^{d} \mathcal{R}^{2} .
\end{aligned}
$$

Thus, at this level one can write a two parameter action containing the above three terms. In general, the conformal mode of this action will not be a Galileon, it would contain other high derivative terms. To get the Galileon we would need to tune the two free parameters to one another in such a way that the term,

$$
\begin{gathered}
4 \int_{\mathcal{M}_{4}} \varepsilon_{a b c d} \Omega_{K}^{a} \wedge E^{b} \wedge E^{c} \wedge E^{d} \Omega_{K} \\
=\int_{\mathcal{M}_{4}} \varepsilon_{a b c d} E^{a} \wedge E^{b} \wedge E^{c} \wedge E^{d} \Omega_{K}^{2},
\end{gathered}
$$

has a vanishing coefficient. This constraint reduces the number of free parameters to one. Furthermore, using IHC and adopting the unitary gauge, $\pi=0$, we can get the metric form of the sought action,

$$
\begin{aligned}
\mathcal{A}_{2}= & \int d^{4} x \sqrt{g}\left[\left(R_{\mu \nu \rho \sigma} R^{\mu \nu \rho \sigma}-4\left[R^{2}\right]+R^{2}\right)\right. \\
& \left.+\alpha W_{\mu \nu \rho \sigma} W^{\mu \nu \rho \sigma}\right] .
\end{aligned}
$$

The term in the parenthesis is the GB term, it is a total derivative in $4 \mathrm{D}$, and its integral is the Euler characteristics of the corresponding manifold. The one parameter freedom in (3.21) enables us to add the square of the Weyl tensor that has a trivial conformal structure, and therefore its coefficient, $\alpha$, is not fixed by our procedure.

At the level of six derivatives there are five independent terms that by naive counting of derivatives and fields could potentially reduce to conformal Galileons,

$$
\begin{aligned}
& \int_{\mathcal{M}_{4}} \varepsilon_{a b c d} \mathcal{R}^{a b} \wedge \mathcal{R}^{c d} \mathcal{R}, \quad \int_{\mathcal{M}_{4}} \varepsilon_{a b c d} \mathcal{R}^{a b} \wedge \mathcal{R}^{c} \wedge \mathcal{R}^{d}, \\
& \int_{\mathcal{M}_{4}} \varepsilon_{a b c d} \mathcal{R}^{a} \wedge \mathcal{R}^{b} \wedge \mathcal{R}^{c} \wedge E^{d}, \\
& \int_{\mathcal{M}_{4}} \varepsilon_{a b c d} \mathcal{R}^{a} \wedge \mathcal{R}^{b} \wedge E^{c} \wedge E^{d} \mathcal{R}, \\
& \int_{\mathcal{M}_{4}} \varepsilon_{a b c d} E^{a} \wedge E^{b} \wedge E^{c} \wedge E^{d} \mathcal{R}^{3} .
\end{aligned}
$$

The action containing the above five terms has four independent parameters, besides its overall multiplier. Requiring that the action for the conformal mode reduces to a conformal Galileon, we get two constraints on the four parameters, ensuring that the following two terms have zero coefficients:

$$
\begin{gathered}
\int_{\mathcal{M}_{4}} \varepsilon_{a b c d} \Omega_{K}^{a} \wedge \Omega_{K}^{b} \wedge E^{c} \wedge E^{d} \Omega_{K}, \\
\int_{\mathcal{M}_{4}} \varepsilon_{a b c d} \Omega_{K}^{a} \wedge E^{b} \wedge E^{c} \wedge E^{d} \Omega_{K}^{2} .
\end{gathered}
$$

Moreover, there are terms containing two powers of the curvature and two covariant derivatives-schematically $D \mathcal{R} D \mathcal{R}$ and implying various contractions-which would in general give more derivatives than the conformal Galileons have; hence we do not include them by finetuning their coefficients to zero. Furthermore, there are other terms with two covariant derivatives and two powers of curvature invariants arranged so that they would vanish if restricted to the conformal mode, e.g., $D W D \mathcal{R}$; such terms would not modify the action for the conformal mode as a stand alone field, and will be included in the full effective theory. They would give new (super)-Planckian mass poles in the propagators (see more discussions on this point in the next section).

Thus, we end up with a two parameter action at the level of six derivatives, which in the metric form and in the unitary gauge can be written as follows:

$$
\begin{aligned}
\mathcal{A}_{3}= & \int d^{4} x \sqrt{g}\left[-R_{\alpha \beta \mu \nu} R^{\alpha \beta \mu \nu} R+12 R_{\mu \nu}^{\alpha \beta} R_{\alpha}^{\mu} R_{\beta}^{\nu}+24\left[R^{3}\right]\right. \\
& -24 R\left[R^{2}\right]+4 R^{3}+\beta_{1} W_{\alpha \beta \mu \nu} W^{\alpha \beta \mu \nu} R \\
& \left.+\beta_{2} W_{\mu \nu}^{\alpha \beta} R_{\alpha}^{\mu} R_{\beta}^{\nu}\right] .
\end{aligned}
$$

As before, the terms proportional to the Weyl tensor are not uniquely determined by our procedure; hence $\beta_{1}$ and $\beta_{2}$ are arbitrary real parameters. This gives a gravitationally dressed action for the $A_{3}$ conformal Galileon.

Last but not least, at the level of eight derivatives, there are six independent invariants which by naive counting of derivatives and fields could potentially reduce to conformal Galileons,

$$
\begin{aligned}
& \int_{\mathcal{M}_{4}} \varepsilon_{a b c d} \mathcal{R}^{a b} \wedge \mathcal{R}^{c d} \mathcal{R}^{2}, \quad \int_{\mathcal{M}_{4}} \varepsilon_{a b c d} \mathcal{R}^{a b} \wedge \mathcal{R}^{c} \wedge \mathcal{R}^{d} \mathcal{R} \\
& \int_{\mathcal{M}_{4}} \varepsilon_{a b c d} \mathcal{R}^{a} \wedge \mathcal{R}^{b} \wedge \mathcal{R}^{c} \wedge \mathcal{R}^{d}, \\
& \int_{\mathcal{M}_{4}} \varepsilon_{a b c d} \mathcal{R}^{a} \wedge \mathcal{R}^{b} \wedge \mathcal{R}^{c} \wedge E^{d} \mathcal{R} \\
& \int_{\mathcal{M}_{4}} \varepsilon_{a b c d} \mathcal{R}^{a} \wedge \mathcal{R}^{b} \wedge E^{c} \wedge E^{d} \mathcal{R}^{2}, \\
& \int_{\mathcal{M}_{4}} \varepsilon_{a b c d} E^{a} \wedge E^{b} \wedge E^{c} \wedge E^{d} \mathcal{R}^{4},
\end{aligned}
$$


requiring five independent parameters in the initial action. In addition to these terms there are ones containing three powers of the curvature and two powers of a covariant derivative, or two powers of the curvature and four powers of the covariant derivative. Some of these terms will be nonzero in the conformal limit (i.e., in the limit when only the conformal mode is kept) and would have more derivatives than present in the conformal Galileons-we do not include such terms by tuning their coefficients to zero. We will, however, include in the full effective Lagrangian the terms that vanish when restricted to the conformal mode. Therefore, the full Lagrangian would still reduce to the conformal Galileon Lagrangian in the conformal limit (see next section).

Furthermore, we impose three constraints on the parameters to guarantee the absence of following terms:

$$
\begin{gathered}
\int_{\mathcal{M}_{4}} \varepsilon_{a b c d} \Omega_{K}^{a} \wedge \Omega_{K}^{b} \wedge \Omega_{K}^{c} \wedge E^{d} \Omega_{K}, \\
\int_{\mathcal{M}_{4}} \varepsilon_{a b c d} \Omega_{K}^{a} \wedge \Omega_{K}^{b} \wedge E^{c} \wedge E^{d} \Omega_{K}^{2}, \\
\int_{\mathcal{M}_{4}} \varepsilon_{a b c d} \Omega_{K}^{a} \wedge E^{b} \wedge E^{c} \wedge E^{d} \Omega_{K}^{3},
\end{gathered}
$$

$$
\begin{aligned}
\mathcal{A}_{2}^{W Z} \equiv & \int_{\mathcal{M}_{5}} \varepsilon_{a b c d} \Omega_{D} \wedge \mathcal{R}^{a b} \wedge \mathcal{R}^{c d} \\
= & \int_{\mathcal{M}_{4}} \varepsilon_{a b c d}\left(8 E^{a} \wedge E^{b} \wedge D \xi^{c} \wedge D \xi^{d}-\frac{32}{3} \xi^{2} E^{a}\right. \\
& \wedge E^{b} \wedge E^{c} \wedge D \xi^{d}+4 \xi^{4} E^{a} \wedge E^{b} \wedge E^{c} \wedge E^{d} \\
& +8 E^{a} \wedge D \xi^{b} \wedge R^{c d}-4 \xi^{2} E^{a} \wedge E^{b} \wedge R^{c d} \\
& \left.+\pi R^{a b} \wedge R^{c d}\right) .
\end{aligned}
$$

The equation of motion for $\xi$ gives IHC; substituting it into $\mathcal{A}_{2}^{W Z}$ we recover the effective action for the scale anomaly derived in [26] (see also [27], where the a-theorem has been proven and $[30,37]$, where it was derived from a different approach),

$$
\begin{aligned}
\mathcal{A}_{2}^{W Z} & \simeq \int d^{4} x \sqrt{g}\left[-\pi\left(R_{\mu \nu \rho \sigma} R^{\mu \nu \rho \sigma}-4\left[R^{2}\right]+R^{2}\right)\right. \\
& \left.+4 \partial^{\mu} \pi \partial^{\nu} \pi\left(R_{\mu \nu}-\frac{1}{2} g_{\mu \nu} R\right)+4(\partial \pi)^{2} \square \pi+2(\partial \pi)^{4}\right] .
\end{aligned}
$$

The latter action, unlike $\mathcal{A}_{\text {coset }}\left(g e^{2 \pi}\right)$, is not Weyl invariant. Its Weyl transformation gives a functional the variation of which, taken at $\pi=0$, gives the trace anomaly. ${ }^{4}$

Thus, the total action of the theory is $\mathcal{A}_{\text {total }}$ in which the $\pi$ field should be restored via the substitution, $g \rightarrow g e^{2 \pi}$, plus the anomalous action $\mathcal{A}_{2}^{W Z}$,

$$
\begin{aligned}
\mathcal{A}_{4}= & \int d^{4} x \sqrt{g}\left(\frac{1}{6} R_{\mu \nu \rho \sigma} R^{\mu \nu \rho \sigma} R^{2}-4 R_{\mu \nu}{ }^{\alpha \beta} R_{\alpha}^{\mu} R_{\beta}^{\nu} R-6\left[R^{4}\right]\right. \\
& +3\left[R^{2}\right]^{2}+\frac{8}{3}\left[R^{3}\right] R-\frac{1}{27} R^{4}+\gamma_{1} W_{\mu \nu \rho \sigma} W^{\mu \nu \rho \sigma} R^{2} \\
& \left.+\gamma_{2} W_{\mu \nu}^{\alpha \beta} R_{\alpha}^{\mu} R_{\beta}^{\nu} R\right) .
\end{aligned}
$$

As before, there are two arbitrary real parameters, $\gamma_{1}$ and $\gamma_{2}$ not fixed by our procedure.

To summarize so far the total coset action is

$$
\mathcal{A}_{\text {coset }}=c_{0} \mathcal{A}_{0}+c_{1} \mathcal{A}_{1}+c_{2} \mathcal{A}_{2}+c_{3} \mathcal{A}_{3}+c_{4} \mathcal{A}_{4}
$$

with $c^{\prime} s$ being real dimensionful coefficients, $c_{0}$ setting the vacuum energy density and $c_{1}$ defining the Planck scale square. The initial Weyl invariance of the action, which so far was gauge fixed for simplicity, can easily be restored by a substitution, $g \rightarrow g e^{2 \pi}$. While in the classical theory such field transformations are harmless, this is not the case in the full quantum theory due to the well-known scale anomaly.

In that regard we note that, $(3.21)$ is not the only term in the second order in curvature; one can write a WessZumino term,

$$
\mathcal{A}_{\text {total }}=\mathcal{A}_{\text {coset }}\left(g e^{2 \pi}\right)+c_{W Z} \mathcal{A}_{2}^{W Z}(g, \pi),
$$

with $\mathcal{A}_{\text {coset }}$ defined in (3.24). The value of the constant $c_{W Z}$ is in general determined by the number of degrees of freedom coupled to gravity, and since we have not introduced any matter degrees of freedom in our case it's only $\pi$ that contributes to $c_{W Z}$. As noted above, the $\pi$ field can no longer be gauged away, because of the anomalous term. If one were to introduce additional fields in the above action, they would couple to gravity via the Weyl invariant couplings; i.e., they would couple to $g e^{2 \pi}$.

The flat space conformal Gallileons can be recovered from $\mathcal{A}_{\text {total }}$ via the substitution $g_{\mu \nu}=\eta_{\mu \nu}$ : the term $\mathcal{A}_{\text {coset }}\left(\eta e^{2 \pi}\right)$ produces a weighted sum of the $A_{0}, A_{1}, A_{3}$, $A_{4}$ conformal Galileons of the previous subsection, while $\mathcal{A}_{2}^{W Z}(\eta, \pi)$ yields the $A_{2}$ conformal Galileon, which was obtained as a WZ term in the flat space case [23].

\footnotetext{
${ }^{4}$ We note that the analogous considerations in $2 \mathrm{D}$ would have given a $3 \mathrm{D}$ WZ term,

$$
\int_{\mathcal{M}_{3}} \varepsilon_{a b} \Omega_{D} \wedge \mathcal{R}^{a b} \simeq \int d^{2} x \sqrt{g}\left[-\pi R-(\partial \pi)^{2}\right],
$$

which reduces to the $2 \mathrm{D}$ Polyakov action.
} 


\section{EFFECTIVE FIELD THEORY AND COSMOLOGY}

The key property of the action (3.27) is that the equation of motion-when restricted to the conformal modehas no more than two derivatives acting on each field, and hence, no new degrees of freedom emerge in the conformal sector.

This is important for cosmology, where the background evolution of a homogeneous and isotropic universe is described by the conformal mode, the scale factor.

However, the existence of the higher powers of the curvatures in the action suggests that the tensor mode propagator will in general have additional poles on curved backgrounds. Such poles, in a fundamentally Lorentz invariant theory, describe ghosts that invalidate the theory, unless the mass of these modes are above the cutoff of the effective field theory (EFT) that (3.27) is part of.

Indeed, the potentially problematic modes in (3.27) have in general a super-Planckian masses as long as the background curvatures are sub-Planckian [this feature is not necessarily specific to the choice of the coefficients between the various terms in (3.27)]. To see this, consider perturbations $h_{\mu \nu}=g_{\mu \nu}-g_{\mu \nu}^{b}$, where $g^{b}$ stands for the background metric. For simplicity, we will focus only on the $R^{3}$ terms, ignore all the tensorial indices, and assume that the background curvature, $R_{b}$, is constant. Then, in the leading order the Lagrangian for small perturbations within any locally flat small neighborhood of a space-time point would take the following schematic form:

$$
\mathcal{L}=h \partial^{2} h+\frac{c R_{b}}{M_{p}^{4}}\left(h \partial^{4} h+R_{b} h \partial^{2} h\right) .
$$

The inverse of the propagator for $h$ would read, $p^{2}(1+$ $\left.\left(c R_{b}^{2} / M_{p}^{4}\right)-\left(c R_{b} p^{2} / M_{p}^{4}\right)\right)$, which guarantees a massless and massive poles in the propagator. In a fundamentally Lorentz invariant theory that we are dealing with the massive pole is necessarily a ghost if the massless one is not. For reasonable values of the parameter $c \sim \mathcal{O}(1)$, and for a sub-Planckian background, $R_{b} \ll M_{p}^{2}$, the ghost mass is super-Planckian, $M_{\text {ghost }}^{2} \simeq M_{p}^{2}\left(M_{p}^{2} / c R_{b}\right)$, and this is outside the effective field theory regime.

On the other hand, if the action (3.27) is just part of an EFT with an infinite number of other terms of growing dimensionality, then the additional terms may spoil the key property of the conformal mode in (3.27) - the facts that this mode is a conformal Galileon. There is a way to deal with this issue as discussed below.

Since the higher order conformal Galileons do not exist, the conformal structure would be maintained only if all the additional terms in EFT vanish for the conformal mode. One way to realize this is to have all the higher order terms be proportional to at least one power of the Weyl tensor. ${ }^{5}$ In this case, the total effective field theory action would take the form,

$\mathcal{A}_{\mathrm{EFT}}=\mathcal{A}_{\mathrm{total}}+\int d^{4} x \sqrt{\bar{g}} \sum_{k=1}^{\infty} \sum_{l=0}^{\infty} \sum_{m=1}^{\infty} b_{k l m} W^{k} \bar{D}^{l} \bar{R}^{m}$,

where $b_{k l m}$ are some coefficients, $\bar{g}=g e^{2 \pi}, W^{k}$ denotes powers of the Weyl tensor, and $\bar{R}$ and $\bar{D}$ denote respectively the Riemann tensor and covariant derivative for $\bar{g}$, with all possible contractions done by the inverse of the metric $\bar{g}$. Terms such as $W \bar{D}^{2} \bar{R}$ would introduce new poles on the flat space, but their masses are (super)Planckian for reasonable values of the parameters in front of such terms.

In each order of EFT in (4.2) there could in general be other terms with more derivatives, such as, $\bar{R} \bar{D}^{2} \bar{R}$, or $\bar{R}^{2} \bar{D}^{2} \bar{R}$, and so on; the coefficients of such terms have been set to zero to guarantee the properties of the conformal sector that we desired. We are not aware of a universal principle that would guarantee such cancellations in the full quantum theory. Such a principle could have emerged due to a theory that would complete the present one at and above the Planck scale. Until that theory is known, our procedure should be regarded as a order-by-order finetuning of the coefficients of counterterms to render the renormalized EFT action free of the higher derivative terms, when it reduced to the theory of the conformal mode only.

The conformal mode is not a physical propagating mode in the EH action. Moreover, its kinetic term has a ghost sign. Nevertheless, it is the mode that describes evolution of the Friedmann-Lemaitre-Robertson-Walker (FLRW) universe. Making sure that no extra derivatives emerge in this sector, i.e., that no Ostrogradski instabilities appear for the conformal mode, is the first stepping stone toward a potentially viable cosmology in any theory with additional terms in the action.

Equipped with the above knowledge we can briefly consider cosmology in a minisuperspace approximation simply to see what novel features might be introduced by the higher curvature terms, and how those novelties play out in the context of EFT. In what follows in this section we set $\pi=0$ as an ansatz, and assume the FLRW metric,

$$
\begin{aligned}
g_{\mu \nu}^{F L R W} d x^{\mu} d x^{\nu}= & \frac{1}{n^{2}(t)} d t^{2}-\frac{a^{2}(t)}{1-k r^{2}} d r^{2}-a^{2}(t) r^{2} d \theta^{2} \\
& -a^{2}(t) r^{2} \sin ^{2} \theta d \phi^{2} .
\end{aligned}
$$

These substitutions nullify all the terms in the EFT action (4.2) that are proportional to the Weyl tensor. Furthermore,

\footnotetext{
${ }^{5}$ The trace of the variation of the Weyl tensor is proportional to the Weyl tensor, so one power on $W$ is already acceptable for our goals.
} 
all the curvature invariants are straightforwardly expressible in terms of the scale factor, $a$, and the inverse lapse, $n$. What remains of the full EFT action (4.2) is the following expression:

$$
\begin{aligned}
- & \frac{1}{12} \int d^{4} x \sqrt{g^{F L R W}} R\left(g^{F L R W}\right)-\frac{1}{24 \beta} \mathcal{A}_{3}\left(g^{F L R W}\right) \\
& -\frac{1}{48 \gamma} \mathcal{A}_{4}\left(g^{F L R W}\right)
\end{aligned}
$$

where we also set the cosmological constant to zero, and renamed the arbitrary coefficients in front of the cubic and quartic order terms as $\beta$ and $\gamma$. The respective minisuperspace Lagrangian reads (up to a total derivative)

$$
\begin{aligned}
\frac{V}{2} & {\left[\frac{a}{n}\left(k-n^{2} \dot{a}^{2}\right)-\frac{1}{a^{3} n \beta}\left(k+n^{2} \dot{a}^{2}\right)^{3}\right.} \\
& +\frac{6}{a^{3} \beta}\left(k^{2} \dot{a}^{2} n+\frac{2}{3} k \dot{a}^{4} n^{3}+\frac{1}{5} \dot{a}^{6} n^{5}\right)+\frac{1}{n a^{5} \gamma}\left(k+n^{2} \dot{a}^{2}\right)^{4} \\
& \left.-\frac{8}{a^{5} \gamma}\left(k^{3} n \dot{a}^{2}+k^{2} n^{3} \dot{a}^{4}+\frac{3}{5} k n^{5} \dot{a}^{6}+\frac{1}{7} n^{7} \dot{a}^{8}\right)\right]
\end{aligned}
$$

Here $V$ stands for the volume of the space: $V=2 \pi^{2}$ for $k=1, V=\infty$ for $k=0$ and $k=-1$. By construction, there are no second and higher time derivatives of the scale factor appearing in the Lagrangian above. Furthermore, let us introduce the notations,

$$
H \equiv \frac{n \dot{a}}{a}, \quad y \equiv H^{2}+\frac{k}{a^{2}(t)} .
$$

Then, the modified Friedmann equation for an empty space-time reads

$$
y-\frac{1}{\beta} y^{3}+\frac{1}{\gamma} y^{4}=0
$$

The cubic and quartic terms in $y$ are suppressed by the respective powers of the cutoff scale (Planck scale). Thus, these terms would modify conventional solutions of the ordinary Friedmann equation by small corrections, as long as the physical scales involved in those solution are significantly lower than the Planck mass scale.

Note however that there are new solutions to the modified Friedmann equation (4.7) which do not exist for the conventional equation. Such solutions could be looked for by finding the zeros of the spacial quartic polynomial in (4.7). To see explicitly some of these solutions let us drop the quartic term in $y$ by taking the limit, $\gamma \rightarrow \infty$. Then, putting $k=1$ and $n=1$ one finds a Starobinski-like solution for a closed universe, which in this case describes a contracting and then reexpanding universe,

$$
a(t)=\frac{1}{\beta^{1 / 4}} \cosh \left(\beta^{1 / 4} t\right) .
$$

There is a similar solution for a spatially flat, as well as open universes, all three representing the de Sitter spacetime. Furthermore, there is also a static solution corresponding to the negative root of the quadratic equation, $y^{2}=\beta$, with an open spatial section, $k=-1$, and a constant scale factor $a=1 / \beta^{1 / 4}$. Weather any of these solutions can be stable with respect to small perturbations is an interesting open question. We only point out that all these solutions invoke curvatures at the cutoff of EFT and are likely to be strongly modified if one were to include other derivative terms in the same order that do not reduce to conformal Galileons. Therefore, the above solutions, and their extensions, require certain fine-tunings of the parameters, as it was done in (4.2).

\section{LOWER DIMENSIONAL DESCENDANTS OF LOVELOCKS}

In this section we will rederive the coset action from the higher dimensional Lovelock terms, by using certain identities $[38,39]$. This method allows us to see familiar results from a different perspective.

\section{A. 3D example}

In [22] we showed that $3 D$ conformal Galileon could be viewed as the Stuickelberg field restoring the local Weyl symmetry in NMG [24], and its extension [25]. Here, we will obtain the same 3D action by dimensionally reducing higher dimensional Lovelock terms. The next subsection will deal with the $4 \mathrm{D}$ case.

Let us begin with the following four Lovelock expressions in space-time of dimensionality $n \geq 3$, which will be relevant for our construction in $3 D$,

$$
\begin{gathered}
\mathcal{A}_{0}^{n D}=-\int_{\mathcal{M}_{n}} \varepsilon_{a_{1} \cdots a_{n}} E^{a_{1}} \wedge \cdots \wedge E^{a_{n}}, \\
\mathcal{A}_{1}^{n D}=-\int_{\mathcal{M}_{n}} \varepsilon_{a_{1} \cdots a_{n}} E^{a_{1}} \wedge \cdots \wedge E^{a_{n-2}} \wedge \mathcal{R}^{a_{n-1} a_{n}}, \\
\mathcal{A}_{2}^{n D}=-\int_{\mathcal{M}_{n}} \varepsilon_{a_{1} \cdots a_{n}} E^{a_{1}} \wedge \cdots \wedge E^{a_{n-4}} \\
\wedge \mathcal{R}^{a_{n-3} a_{n-2}} \wedge \mathcal{R}^{a_{n-1} a_{n}}, \\
\mathcal{A}_{3}^{n D}=-\int_{\mathcal{M}_{n}} \varepsilon_{a_{1} \cdots a_{n}} E^{a_{1}} \wedge \cdots \wedge E^{a_{n-6}} \wedge \mathcal{R}^{a_{n-5} a_{n-4}} \\
\wedge \mathcal{R}^{a_{n-3} a_{n-2}} \wedge \mathcal{R}^{a_{n-1} a_{n}} .
\end{gathered}
$$

These also happen to be the terms of the conformal/ Poincaré coset; hence they realize nonlinearly the special conformal and dilatation symmetries. Moreover, all of these 
terms have the desired conformal structure; i.e., the conformal mode is a conformal Galileon in $n$ dimensional space-time. The IHC is a solution to the corresponding equations of motion, so we will use $\xi^{\mu}=-1 / 2 e^{-\pi} \partial^{\mu} \pi$. [It is convenient to work in the unitary gauge $(\pi=0)$, and if needed, one can recover the $\pi$ interactions by making a field redefinition $g_{\mu \nu} \rightarrow e^{2 \pi} g_{\mu \nu}$ in the unitary gauge classical action.]

Let us rewrite the above expressions in the metric form,

$$
\begin{gathered}
\mathcal{A}_{0}^{n D}=n ! \\
\mathcal{A}_{1}^{n D}=\int d^{n} x \sqrt{g}\left[\frac{1}{2}(n-2) ! \delta_{\nu_{1} \nu_{2}}^{\mu_{1} \mu_{2}} R^{\nu_{1} \nu_{2}} \mu_{1} \mu_{2}\right] \\
\mathcal{A}_{2}^{n D}=\int d^{n} x \sqrt{g}\left[\frac{1}{2^{2}}(n-4) ! \delta_{\nu_{1} \cdots \nu_{4}}^{\mu_{1} \cdots \mu_{4}} R^{\nu_{1} \nu_{2}} \mu_{1} \mu_{2} R^{\nu_{3} \nu_{4}} \mu_{3} \mu_{4}\right] \\
\mathcal{A}_{3}^{n D}=\int d^{n} x \sqrt{g} \\
\times\left[\frac{1}{2^{3}}(n-6) ! \delta_{\nu_{1} \cdots \nu_{6}}^{\mu_{1} \cdots \mu_{6}} R^{\nu_{1} \nu_{2}} \mu_{\mu_{1} \mu_{2}} R^{\nu_{3} \nu_{4}} \mu_{3} \mu_{4} R^{\nu_{5} \nu_{6}}{ }_{\mu_{5} \mu_{6}}\right] .
\end{gathered}
$$

Note that $\mathcal{A}_{0}^{n D}$ and $\mathcal{A}_{1}^{n D}$ are both regular for $n=3$, the problems arise with $\mathcal{A}_{2}^{n D}$ and $\mathcal{A}_{3}^{n D}$, both of which contain products $\infty \times 0$. The infinity comes from the factorial and zero from the generalized Kronecker symbol (the latter is zero in $n=3$ because it contains more than three antisymmetrised indices). One needs to regularize the above expressions to make sense of them. Regularization of $\mathcal{A}_{2}^{n D}$ is relatively easy, one can assume that for $n<4$ the Weyl tensor $W^{\mu \nu}{ }_{\alpha \beta}$ vanishes identically; obviously, the Weyl tensor vanishes for $n=3$, but the assumption is that it also vanishes for $3 \leq n<4$ as we analytically continue the parameter $n$. This enables us to express the Riemann tensor for $3 \leq n<4$ as follows:

$$
\begin{aligned}
R_{\alpha \beta}^{\mu \nu}= & \frac{1}{n-2}\left(R_{\alpha}^{\mu} \delta_{\beta}^{\nu}-R_{\beta}^{\mu} \delta_{\alpha}^{\nu}+\delta_{\alpha}^{\mu} R_{\beta}^{\nu}-\delta_{\beta}^{\mu} R_{\alpha}^{\nu}\right) \\
& -\frac{1}{(n-2)(n-1)} R\left(\delta_{\alpha}^{\mu} \delta_{\beta}^{\nu}-\delta_{\beta}^{\mu} \delta_{\alpha}^{\nu}\right) .
\end{aligned}
$$

We can now substitute this relation into the Lovelock action (5.7) to express the latter as follows:

$\mathcal{A}_{2}^{n D}=\int d^{n} x \sqrt{g}\left[-4 \frac{(n-3) !}{n-2}\left(\left[R^{2}\right]-\frac{n}{4(n-1)} R^{2}\right)\right]$.

This expression is regular for $n=3$, and upon this substitution gives the action of NMG [24].

The above procedure-referred as the method of higher dimensional reduction (HDR) - defines formal analytic continuation of Lovelocks from $n>3$, down to $n=3$. We will use now HDR for other terms. ${ }^{6}$

To regularize $\mathcal{A}_{3}^{n D}$ we have to take more steps down the ladder of dimensionalities. First we regularize (5.8) in $5 D$, then in $4 D$, and only after in $3 D$; hence we have a cascade of regularizations,

$$
6 D \rightarrow 5 D \rightarrow 4 D \rightarrow 3 D .
$$

The first step in this cascade consists of adopting the following identity for $n<6$ :

$$
\delta_{\nu_{1} \cdots \nu_{6}}^{\mu_{1} \cdots \mu_{6}} W^{\nu_{1} \nu_{2}}{ }_{\mu_{1} \mu_{2}} W^{\nu_{3} \nu_{4}}{ }_{\mu_{3} \mu_{4}} W^{\nu_{5} \nu_{6}}{ }_{\mu_{5} \mu_{6}}=0 .
$$

As soon as this is used, the action (5.8) for $\mathcal{A}_{3}^{n D}$ takes the form,

$$
\begin{aligned}
& \int d^{n} x \sqrt{g} \frac{(n-5) !}{n-2}\left[\frac{3(n+2)}{n-1} R_{\alpha \beta}{ }^{\mu \nu} R_{\mu \nu}{ }^{\alpha \beta} R\right. \\
& +24 R_{\mu \nu}{ }^{\alpha \beta} R_{\beta \rho}{ }^{\mu \nu} R_{\alpha}^{\rho}+\frac{24 n}{n-2} R_{\mu \nu}{ }^{\alpha \beta} R_{\alpha}^{\mu} R_{\beta}^{\nu} \\
& +\frac{16 n(n-1)}{(n-2)^{2}}\left[R^{3}\right]-\frac{12\left(n^{3}-2 n^{2}+6 n-8\right)}{(n-1)(n-2)^{2}}\left[R^{2}\right] R \\
& \left.+\frac{n^{4}-3 n^{3}+10 n^{2}+4 n-24}{(n-1)^{2}(n-2)^{2}} R^{3}\right],
\end{aligned}
$$

which is now regular in $5 D$. The next step is to descend to $4 D$. The expression in the square brackets is zero in $n=4$, while the overall multiplier is diverging; hence further regularization is needed. The latter can be achieved by means of the following identity:

$$
R_{\nu}^{\mu} \delta_{\mu \alpha \beta \gamma \delta}^{\nu \lambda \rho \omega} W_{\lambda \rho}^{\alpha \beta} W_{\sigma \omega}{ }^{\gamma \delta}=R_{\nu}^{\mu} \cdot 0=0 .
$$

Using the above, the regularized $\mathcal{A}_{3}^{n D}$ for $n<5$ can be written as follows:

$$
\begin{aligned}
\mathcal{A}_{3}^{n D}= & \int d^{n} x \sqrt{g} \frac{(n-4) !}{n-2}\left[-\frac{3}{n-1} R_{\alpha \beta \mu \nu} R^{\alpha \beta \mu \nu} R\right. \\
& +\frac{24}{n-2} R_{\mu \nu}^{\alpha \beta} R_{\alpha}^{\mu} R_{\beta}^{\nu}+\frac{16 n}{(n-2)^{2}}\left[R^{3}\right] \\
& \left.-\frac{12 n^{2}}{(n-1)(n-2)^{2}}\left[R^{2}\right] R+\frac{n\left(n^{2}+n+2\right)}{(n-1)^{2}(n-2)^{2}} R^{3}\right] .
\end{aligned}
$$

\footnotetext{
${ }^{6} \mathrm{An}$ equivalent method of regularization in this case is to subtract the term $\delta_{\nu_{1} \cdots \nu_{4}}^{\mu_{1} \cdots \mu_{4}} W^{\nu_{1} \nu_{2}}{ }_{\mu_{1} \mu_{2}} W^{\nu_{3} \nu_{4}}{ }_{\mu_{3} \mu_{4}}$ from the original $n$ dimensional action with an appropriate coefficient to eliminate the maximal tensorial structure (in this case $R_{\alpha \beta \mu \nu} R^{\alpha \beta \mu \nu}$ ). The two methods are equivalent. It turns out that in more complicated cases the latter method is more convenient; also the subtraction does explain the emergence of an additional massive spin- 2 mode in the lower dimensional actions, while this mode does not exist in the starting Lovelock action.
} 
It is straightforward to check that the action is regular for $n=4$. For the last step of the cascade we use (5.9) to get a perfectly regular expression for $n<4$,

$$
\begin{aligned}
\mathcal{A}_{3}^{n D}= & \int d^{n} x \sqrt{g} \frac{(n-3) !}{(n-2)^{3}} \\
& \times\left[16\left[R^{3}\right]-\frac{12 n}{n-1}\left[R^{2}\right] R+\frac{n^{2}+4 n-4}{(n-1)^{2}} R^{3}\right] .
\end{aligned}
$$

Substituting $n=3$ in the above expression we get the action that extends NMG to the cubic order [25]. The fact that there is a connection between the Lovelock terms and NMG was already shown in Ref. [25] on the basis of equivalence between their dual CFTs (see also $[40,41]$ ). Our method is complementary.

\section{B. 4D theory}

By now one should perhaps expect that the gravitationally dressed conformal Galileons are certain descendants of higher dimensional Lovelock terms. This point of view will be reinforced below by obtaining the coset action of Sec. III from the Lovelock terms, using the method of HDR.

Let us first briefly summarize the rules of HDR:

(i) To descend from $m$ dimensions down to $(m-1)$ dimensions we use identities involving the symbol $\delta_{\nu_{1} \cdots \nu_{m}}^{\mu_{1} \cdots \mu_{m}}$ contracted with curvature tensors.

(ii) Each identity must involve at least one Weyl tensor; otherwise the identity should not use the Riemann tensor.

(iii) At every step there are a finite number of the identities to be used, and usually one needs all of them.

After using the identities one gets $(m-1) \times L^{k}$, where $L^{k}$ is the analogue of the $k$ th order Lovelock in $(m-1)<2 k$ dimensions (i.e., it has the same conformal structure as the Lovelock). If the rules described above are not sufficient to extract the factor of $(m-1)$, then the corresponding Lovelock does not have an analogue in $(m-1)$ dimensions (i.e., there are no terms with the conformal structure of the Lovelock). Note that this definition of an "analogue" leaves a room for degeneracy (as we will see below), but the terms we get through the above described procedure are guaranteed to be the most general ones.

Let us now apply HDR in 4D. Among the terms (5.5)(5.8) only $\mathcal{A}_{3}^{n D}$ needs regularization in $4 \mathrm{D}$; however, this was already done in (5.13) en route to the $3 D$ expression. Hence, substituting $n=4$ into (5.13) we get

$$
\begin{aligned}
\mathcal{A}_{3}^{4 D}= & \int d^{4} x \sqrt{g} \frac{1}{2}\left(-R_{\alpha \beta \mu \nu} R^{\alpha \beta \mu \nu} R+12 R_{\mu \nu}{ }^{\alpha \beta} R_{\alpha}^{\mu} R_{\beta}^{\nu}\right. \\
& \left.+16\left[R^{3}\right]-16\left[R^{2}\right] R+\frac{22}{9} R^{3}\right) .
\end{aligned}
$$

This action coincides with $\mathcal{A}_{3}$ that we've derived in (3.22) by using the coset construction. ${ }^{7}$

Let us now see how $\mathcal{A}_{4}$ of (3.23) comes along in this formalism. For this we look at an eight derivative Lovelock term in $n$ dimensions,

$$
\begin{aligned}
\mathcal{A}_{4}^{n D}= & -\int_{\mathcal{M}_{n}} \varepsilon_{a_{1} \cdots a_{n}} E^{a_{1}} \wedge \cdots \wedge E^{a_{n-8}} \wedge \mathcal{R}^{a_{n-7} a_{n-6}} \\
& \wedge \mathcal{R}^{a_{n-5} a_{n-4}} \wedge \mathcal{R}^{a_{n-3} a_{n-2}} \wedge \mathcal{R}^{a_{n-1} a_{n}} .
\end{aligned}
$$

In the unitary gauge and in the metric formulation the above expression becomes

$$
\begin{aligned}
\mathcal{A}_{4}^{n D}= & \int d^{n} x \sqrt{g} \frac{1}{2^{4}}(n-8) ! \delta_{\nu_{1} \cdots \nu_{8}}^{\mu_{1} \cdots \mu_{8}} R^{\nu_{1} \nu_{2}}{ }_{\mu_{1} \mu_{2}} \\
& \times R^{\nu_{3} \nu_{4}}{ }_{\mu_{3} \mu_{4}} R^{\nu_{5} \nu_{6}}{ }_{\mu_{5} \mu_{6}} R^{\nu_{7} \nu_{8}}{ }_{\mu_{7} \mu_{8} .} .
\end{aligned}
$$

Regularization of this expression is a tedious task. We will not fully describe the process but rather give the necessary identities for each step of HDR,

$$
\begin{aligned}
& 8 D \rightarrow 7 D \quad \delta_{\nu_{1} \cdots \nu_{8}}^{\mu_{1} \cdots \mu_{8}} W^{\nu_{1} \nu_{2}}{ }_{\mu_{1} \mu_{2}} W^{\nu_{3} \nu_{4}}{ }_{\mu_{3} \mu_{4}} W^{\nu_{5} \nu_{6}}{ }_{\mu_{5} \mu_{6}} W^{\nu_{7} \nu_{8}}{ }_{\mu_{7} \mu_{8}}=0, \\
& 7 D \rightarrow 6 D \quad \delta_{\nu_{1} \cdots \nu_{7}}^{\mu_{1} \cdots \mu_{7}} W^{\nu_{1} \nu_{2}}{ }_{\mu_{1} \mu_{2}} W^{\nu_{3} \nu_{4}}{ }_{\mu_{3} \mu_{4}} W^{\nu_{5} \nu_{6}}{ }_{\mu_{5} \mu_{6}} R_{\mu_{7}}^{\nu_{7}}=0, \\
& 6 D \rightarrow 5 D \quad \delta_{\nu_{1} \cdots \nu_{6}}^{\mu_{1} \cdots \mu_{6}} W^{\nu_{1} \nu_{2}}{ }_{\mu_{1} \mu_{2}} W^{\nu_{3} \nu_{4}}{ }_{\mu_{3} \mu_{4}} W^{\nu_{5} \nu_{6}}{ }_{\mu_{5} \mu_{6}} R=0, \\
& \delta_{\nu_{1} \cdots \nu_{6}}^{\mu_{1} \cdots \mu_{6}} W^{\nu_{1} \nu_{2}}{ }_{\mu_{1} \mu_{2}} W^{\nu_{3} \nu_{4}}{ }_{\mu_{3} \mu_{4}} R^{\nu_{5}}{ }_{\mu_{5}} R^{\nu_{6}}{ }_{\mu_{6}}=0, \\
& 5 D \rightarrow 4 D \quad \delta_{\nu_{1} \cdots \nu_{5}}^{\mu_{1} \cdots \mu_{5}} W^{\nu_{1} \nu_{2}}{ }_{\mu_{1} \mu_{2}} W^{\nu_{3} \nu_{4}}{ }_{\mu_{3} \mu_{4}} R^{\nu_{5}}{ }_{\mu_{5}} R=0, \\
& \delta_{\nu_{1} \cdots \nu_{5}}^{\mu_{1} \cdots \mu_{5}} W^{\nu_{1} \nu_{2}}{ }_{\mu_{1} \mu_{2}} R^{\nu_{3}}{ }_{\mu_{3}} R^{\nu_{4}}{ }_{\mu_{4}} R^{\nu_{5}}{ }_{\mu_{5}}=0 .
\end{aligned}
$$

The result of this lengthy procedure is the regularized action valid for $4 \leq n<5$,

$$
\begin{aligned}
\mathcal{A}_{4}^{n D}= & \int d^{n} x \sqrt{g} \frac{(n-4) !}{(n-1)^{3}(n-2)^{4}} \\
& \times\left[6(n-2)^{2}(n-1) R_{\mu \nu \rho \sigma} R^{\mu \nu \rho \sigma} R^{2}\right. \\
& -96(n-2)(n-1)^{2} R_{\mu \nu}{ }^{\alpha \beta} R_{\alpha}^{\mu} R_{\beta}^{\nu} R-96(n-1)^{3}\left[R^{4}\right] \\
& +48(n-1)^{3}\left[R^{2}\right]^{2}+64(n-2)(n-1)^{2}\left[R^{3}\right] R \\
& -24 n(n-4)(n-1)\left[R^{2}\right] R^{2} \\
& \left.+\left(n^{3}+11 n^{2}-78 n+56\right) R^{4}\right] .
\end{aligned}
$$

Substituting $n=4$ into the above expression we get the $4 D$ action in the unitary gauge,

\footnotetext{
${ }^{7}$ It corresponds to the case of $\beta_{1}=\beta_{2}=0$, but the latter terms can always be added since they do not affect the conformal structure and are part of the degeneracy we mentioned in the previous paragraph.
} 


$$
\begin{aligned}
\mathcal{A}_{4}^{4 D}= & \int d^{4} x \sqrt{g}\left(\frac{1}{6} R_{\mu \nu \rho \sigma} R^{\mu \nu \rho \sigma} R^{2}-4 R_{\mu \nu}{ }^{\alpha \beta} R_{\alpha}^{\mu} R_{\beta}^{\nu} R\right. \\
& \left.-6\left[R^{4}\right]+3\left[R^{2}\right]^{2}+\frac{8}{3}\left[R^{3}\right] R-\frac{1}{27} R^{4}\right) .
\end{aligned}
$$

This coincides with $\mathcal{A}_{4}$ in (3.23), with $\gamma_{1}=\gamma_{2}=0$. This completes the derivation of all the $4 \mathrm{D}$ coset terms from the higher dimensional Lovelock terms.

\section{ACKNOWLEDGMENTS}

We would like to thank Massimo Porrati, Samson Shatashvili, Jim Simons, and Alex Vilenkin for useful discussions. The work on G. G. is supported by the NSF Grant No. PHY-1915219. G. T. is supported by the Simons Foundations "Origins of the Universe" program.
[1] G. R. Dvali, G. Gabadadze, and M. Porrati, 4-D gravity on a brane in 5-D Minkowski space, Phys. Lett. B 485, 208 (2000).

[2] C. de Rham and G. Gabadadze, Generalization of the FierzPauli action, Phys. Rev. D 82, 044020 (2010).

[3] C. de Rham, G. Gabadadze, and A. J. Tolley, Resummation of Massive Gravity, Phys. Rev. Lett. 106, 231101 (2011).

[4] A. Vainshtein and I. Khriplovich, On the zero-mass limit and renormalizability in the theory of massive Yang-Mills field, Yad. Fiz. 13, 198 (1971).

[5] A. I. Vainshtein, To the problem of nonvanishing gravitation mass, Phys. Lett. 39B, 393 (1972).

[6] C. Deffayet, G. Dvali, G. Gabadadze, and A. I. Vainshtein, Nonperturbative continuity in graviton mass versus perturbative discontinuity, Phys. Rev. D 65, 044026 (2002).

[7] N. Arkani-Hamed, H. Georgi, and M. D. Schwartz, Effective field theory for massive gravitons and gravity in theory space, Ann. Phys. (Amsterdam) 305, 96 (2003).

[8] M. A. Luty, M. Porrati, and R. Rattazzi, Strong interactions and stability in the DGP model, J. High Energy Phys. 09 (2003) 029.

[9] A. Adams, N. Arkani-Hamed, S. Dubovsky, A. Nicolis, and R. Rattazzi, Causality, analyticity and an IR obstruction to UV completion, J. High Energy Phys. 10 (2006) 014.

[10] E. Babichev and C. Deffayet, An introduction to the Vainshtein mechanism, Classical Quantum Gravity 30, 184001 (2013).

[11] C. Cheung and G. N. Remmen, Positive signs in massive gravity, J. High Energy Phys. 04 (2016) 002.

[12] C. de Rham, S. Melville, and A. J. Tolley, Improved positivity bounds and massive gravity, J. High Energy Phys. 04 (2018) 083.

[13] C. de Rham, S. Melville, A. J. Tolley, and S. Y. Zhou, Positivity bounds for massive spin-1 and spin-2 fields, J. High Energy Phys. 03 (2019) 182.

[14] J. Bonifacio, K. Hinterbichler, and R. A. Rosen, Constraints on a gravitational Higgs mechanism, Phys. Rev. D 100, 084017 (2019).

[15] H. Ogawa, T. Kobayashi, and K. Koyama, Relativistic stars in a cubic Galileon Universe, Phys. Rev. D 101, 024026 (2020).

[16] A. Nicolis, R. Rattazzi, and E. Trincherini, The Galileon as a local modification of gravity, Phys. Rev. D 79, 064036 (2009).
[17] C. Deffayet, X. Gao, D. A. Steer, and G. Zahariade, From k-essence to generalised Galileons, Phys. Rev. D 84, 064039 (2011).

[18] M. Mirbabayi, A proof of ghost freedom in de RhamGabadadze-Tolley massive gravity, Phys. Rev. D 86, 084006 (2012).

[19] P. Creminelli, A. Nicolis, and E. Trincherini, Galilean genesis: An alternative to inflation, J. Cosmol. Astropart. Phys. 11 (2010) 021.

[20] P. Creminelli, K. Hinterbichler, J. Khoury, A. Nicolis, and E. Trincherini, Subluminal Galilean genesis, J. High Energy Phys. 02 (2013) 006.

[21] D. Pirtskhalava, L. Santoni, E. Trincherini, and P. Uttayarat, Inflation from Minkowski space, J. High Energy Phys. 12 (2014) 151.

[22] G. Gabadadze and G. Tukhashvili, Gravitational dressing of 3D conformal Galileon, Phys. Lett. B 793, 415 (2019).

[23] G. Goon, K. Hinterbichler, A. Joyce, and M. Trodden, Galileons as Wess-Zumino Terms, J. High Energy Phys. 06 (2012) 004.

[24] E. A. Bergshoeff, O. Hohm, and P. K. Townsend, Massive Gravity in Three Dimensions, Phys. Rev. Lett. 102, 201301 (2009).

[25] A. Sinha, On the new massive gravity and AdS/CFT, J. High Energy Phys. 06 (2010) 061.

[26] R. J. Riegert, A nonlocal action for the trace anomaly, Phys. Lett. 134B, 56 (1984).

[27] Z. Komargodski and A. Schwimmer, On renormalization group flows in four dimensions, J. High Energy Phys. 12 (2011) 099.

[28] D. Glavan and C. Lin, Einstein-Gauss-Bonnet Gravity in 4-Dimensional Space-Time, Phys. Rev. Lett. 124, 081301 (2020).

[29] M. Gurses, T. C. Sisman, and B. Tekin, Is there a novel Einstein-Gauss-Bonnet theory in four dimensions?, arXiv: 2004.03390.

[30] J. Bonifacio, K. Hinterbichler, and L. A. Johnson, Amplitudes and 4D Gauss-Bonnet theory, arXiv:2004.10716 [Phys. Rev. D (to be published)].

[31] C. G. Callan, Jr., S. R. Coleman, J. Wess, and B. Zumino, Structure of phenomenological Lagrangians. 2., Phys. Rev. 177, 2247 (1969). 
[32] E. A. Ivanov and V.I. Ogievetsky, The inverse Higgs phenomenon in nonlinear realizations, Teor. Mat. Fiz. 25, 164 (1975).

[33] A. B. Borisov and V. I. Ogievetsky, Theory of dynamical affine and conformal symmetries as the theory of the gravitational field, Teor. Mat. Fiz. 21, 329 (1974) [Theor. Math. Phys. 21, 1179 (1975)].

[34] L. V. Delacrtaz, S. Endlich, A. Monin, R. Penco, and F. Riva, (Re-)inventing the relativistic wheel: Gravity, cosets, and spinning objects, J. High Energy Phys. 11 (2014) 008.

[35] I. N. McArthur, Nonlinear realizations of symmetries and unphysical Goldstone bosons, J. High Energy Phys. 11 (2010) 140.

[36] K. Farnsworth, M. A. Luty, and V. Prilepina, Weyl versus conformal invariance in quantum field theory, J. High Energy Phys. 10 (2017) 170.
[37] P. G. S. Fernandes, P. Carrilho, T. Clifton, and D. J. Mulryne, Derivation of regularized field equations for the Einstein-Gauss-Bonnet theory in four dimensions, Phys. Rev. D 102, 024025 (2020).

[38] B. S. DeWitt, Dynamical theory of groups and fields, Conf. Proc. C 630701, 585 (1964); Les Houches Lect. Notes 13, 585 (1964).

[39] D. Lovelock, Dimensionally dependent identities, Proc. Cambridge Philos. Soc. 68, 345 (1970).

[40] J. Oliva and S. Ray, A new cubic theory of gravity in five dimensions: black hole, Birkhoff's theorem and C-function, Classical Quantum Gravity 27, 225002 (2010).

[41] G. Alkac and D. Olgu Devecioglu, Three dimensional modified gravities as holographic limits of Lancsoz-Lovelock theories, Phys. Lett. B 807, 135597 (2020). 